EPJ manuscript No.

(will be inserted by the editor)

\title{
Transverse momentum distributions of neutral pions from nuclear collisions at $200 A \mathrm{GeV}$
}

\section{WA80 Collaboration} \author{
A. Vinogradov ${ }^{2}$, and G.R. Young ${ }^{3}$ \\ 1 Gesellschaft für Schwerionenforschung, D-64220 Darmstadt, Germany \\ 2 Russian Research Center "Kurchatov Institute", Moscow 123182, Russia \\ 3 Oak Ridge National Laboratory, Oak Ridge, Tennessee 37831, USA \\ ${ }^{4}$ University of Münster, D-48149 Münster, Germany \\ ${ }^{5}$ Lawrence Berkeley Laboratory, Berkeley, California 94720, USA \\ ${ }^{6}$ University of Lund, S-22362 Lund, Sweden \\ ${ }^{7}$ Universiteit Utrecht/NIKHEF, NL-3508 TA Utrecht, The Netherlands \\ 8 KVI, University of Groningen, NL-9747 AA Groningen, The Netherlands \\ ${ }^{9}$ University of Tennessee, Knoxville, Tennessee 37996, USA
}

R. Albrecht ${ }^{1}$, V. Antonenko ${ }^{2}$, T.C. Awes ${ }^{3}$, C. Barlag ${ }^{4}$, M.A. Bloomer ${ }^{5}$, C. Blume ${ }^{4}$, D. Bock ${ }^{4}$, R. Bock ${ }^{1}$, E.M. Bohne ${ }^{4}$, D. Bucher ${ }^{4}$, A. Claussen ${ }^{4}$, G. Clewing ${ }^{4}$, A. Eklund ${ }^{6}$, S. Fokin ${ }^{2}$, A. Franz ${ }^{3}$, S. Garpman ${ }^{6}$, F. Geurts ${ }^{7}$, R. Glasow ${ }^{4}$, H.Å. Gustafsson ${ }^{6}$, H.H. Gutbrod ${ }^{1}$ a , G. Hölker ${ }^{4}$, J. Idh ${ }^{6}$, M. Ippolitov ${ }^{2}$, P. Jacobs ${ }^{5}$, R. Kamermans ${ }^{7}$, K.-H. Kampert ${ }^{4}$ b K. Karadjev ${ }^{2}$, B.W. Kolb ${ }^{1}$, A. Lebedev ${ }^{2}$, H. Löhner ${ }^{8}$, I. Lund ${ }^{8}$, V. Manko ${ }^{2}$, S. Nikolaev ${ }^{2}$, F.E. Obenshain ${ }^{3}$, A. Oskarsson ${ }^{6}$, I. Otterlund $^{6}$, T. Peitzmann ${ }^{4}$, F. Plasil ${ }^{3}$, A.M. Poskanzer ${ }^{5}$, M. Purschke ${ }^{1}$, H.G. Ritter ${ }^{5}$, S. Saini ${ }^{3}$, R. Santo ${ }^{4}$, H.R. Schmidt ${ }^{1}$, K. Söderstrom ${ }^{6}$, S.P. Sørensen ${ }^{3,9}$, P. Stankus ${ }^{3}$, K. Steffens ${ }^{4}$, P. Steinhaeuser ${ }^{1}$, E. Stenlund ${ }^{6}$, D. Stüken ${ }^{4}$, C. Twenhöfel ${ }^{7}$,

Accepted for publication in Eur. Phys. J. C

\begin{abstract}
New results on transverse mass spectra of neutral pions measured at central rapidity are presented for impact parameter selected $200 \mathrm{~A} \cdot \mathrm{GeV} \mathrm{S}+\mathrm{S}$ and $\mathrm{S}+\mathrm{Au}$ collisions. The distributions cover more than 8 orders of magnitude in cross section over the range $0.3 \mathrm{GeV} / c \leq p_{T} \leq 4.0 \mathrm{GeV} / c$. Detailed comparisons to results from pp collisions are made. The spectra from all systems show a clear power-law like shape with similar curvature. Collisions of $\mathrm{S}+\mathrm{Au}$ exhibit a larger mean transverse momentum than pp increasing with centrality. Predictions of string models and by hydrodynamic approaches including collective expansion and decays of short lived resonances are compared to the data and the implications are discussed.
\end{abstract}

\section{Introduction}

Heavy ion reactions at sufficiently high energies are able to create a strongly compressed and highly excited reaction zone which may provide the conditions for the transition to a deconfined state of nuclear matter, the Quark-Gluon Plasma. Direct information on this state and the initial conditions are provided by probes which decouple early in the reaction, like photons and dileptons. Hadrons which interact strongly decouple much later and their transverse momentum spectrum therefore gives information about the condition of the system at freeze out. By combining the information from photons or dileptons and from hadrons and comparing with data from proton-proton interactions it is hoped that the development of the heavy-ion system from the initial state of high density and temperature through freeze out can be reconstructed. In early pp scattering experiments, it has been found that $p_{T}$ spectra of pions in the range

\footnotetext{
a now at SUBATECH, Ecole des Mines, Nantes, France

b now at University of Karlsruhe (TH), D-76021 Karlsruhe
}

up to about $2 \mathrm{GeV} / c$ exhibit a nearly exponential shape with a slope constant of about $160 \mathrm{MeV} / c$. This is at first sight surprising since most of the pions are not produced directly but emerge from heavier resonances with different decay energies. Furthermore, exponential spectra are usually observed in systems, where thermodynamical equilibrium is reached through a sizable number of rescatterings. In a proton-proton collision this is hardly conceivable. Using the bootstrap hypothesis and particular assumptions on the particle mass spectrum, Hagedorn was able to describe the exponential shape of the spectra and the limiting value of the slopes [1]].

Proton-proton experiments at the CERN ISR extended measurements to $p_{T}$ values beyond $2 \mathrm{GeV} / c$ [2, 3, 4 ] and subsequently revealed distinct deviations from an exponential shape approaching a more powerlaw-like behavior. This was interpreted as the onset of "hard" parton scattering [5] and can be treated to some extent by perturbative QCD [6].

Data on hadron-nucleus collisions display further characteristic differences when compared to proton-proton collisions. These are often parametrized by a factor $A^{\alpha\left(p_{T}\right)}$ connecting 
Albrecht et al.: Transverse momentum distributions of neutral pions from nuclear collisions at $200 \mathrm{AGeV}$

$\mathrm{p}+\mathrm{p}$ and $\mathrm{p}+A$ cross sections. At low transverse momenta, $\alpha\left(p_{T}\right)$ turns out to be about 0.7 , which is close to the value of $2 / 3$ expected from a geometrical picture of hadron production from an opaque target nucleus. For larger transverse momenta, $\alpha\left(p_{T}\right)$ increases, which is interpreted as the onset of parton scattering extending over the whole nuclear volume. In this picture, $\alpha\left(p_{T}\right)$ should finally approach the value 1 . The surprising observation of $\alpha>1$ in the experimental spectra at high $p_{T} \gtrsim 3$ $\mathrm{GeV} / c$ (Cronin-effect [7]) was then interpreted as the effect of multiple parton scattering [8,9].

All of the features of proton-proton and hadron-nucleus scattering described above are expected to be present in energetic collisions between heavy nuclei. In addition, due to the nuclear matter being highly excited and compressed over a large volume, new specific thermodynamic or hydrodynamic features are expected to become apparent.

Theoretically, both the description of nucleus-nucleus collisions as a superposition of hadron-hadron collisions [10,11, 12, as well as the assumption of a hot hydrodynamically expanding system of hadrons or partons [13,14], originally proposed by Landau [15] are being pursued.

In both approaches, the approximate exponential shape of the final spectra at low $p_{T}$ results from a convolution or superposition of various basic processes and sequential decays, and cannot easily be associated with a "temperature". At high $p_{T}$, on the other hand, it is expected that the spectra converge to the "hard scattering" shapes observed in proton-proton scattering and successfully described by perturbative QCD.

In order to distinguish between the theoretical approaches and to isolate possible new features of heavy-ion collisions, it is mandatory to investigate the transverse momentum spectra of identified particles over a large range in $p_{T}$ extending in particular to the hard scattering regime.

In general, the invariant differential cross sections of produced particles measured from a given reaction system depend not only on the transverse momentum $p_{T}$, but also on the available center-of-mass ( $\mathrm{cms}$ ) energy $\sqrt{s}$, the rapidity $y$ of the particle, and the associated centrality. A comparison of $p_{T}$ spectra from different collision systems or different data sets therefore has to carefully consider the dependencies on the quantities noted above.

In [16], preliminary results of neutral pion spectra for Sinduced reactions have been presented. In the present paper, the final results on high precision neutral pion $p_{T}$ spectra from collisions of $200 A \cdot \mathrm{GeV}$ sulphur nuclei $(\sqrt{s}=19.4 A \mathrm{GeV})$ with sulphur and gold targets in the range $0.3 \mathrm{GeV} / c \leq p_{T} \leq$ $4.0 \mathrm{GeV} / c$ and $2.1 \leq y \leq 2.9$ for various centralities after a complete reanalysis are shown. They will be compared to data from $\mathrm{p}+\mathrm{p}$ and $\mathrm{p}+\mathrm{A}$ interactions. The production of $\eta$ mesons measured within the same experiment over the $p_{T}$ range of $0.5 \leq p_{T} \leq 3.5 \mathrm{GeV} / c[17]$ and an upper limit on direct photon production [18] have been recently published.

\section{Experiment}

The WA80 experiment at the CERN-SPS (Fig.11) was equipped with a finely segmented electromagnetic spectrometer composed of 3798 lead-glass modules with photomultiplier readout. The lead-glass was arranged into three independently cal-

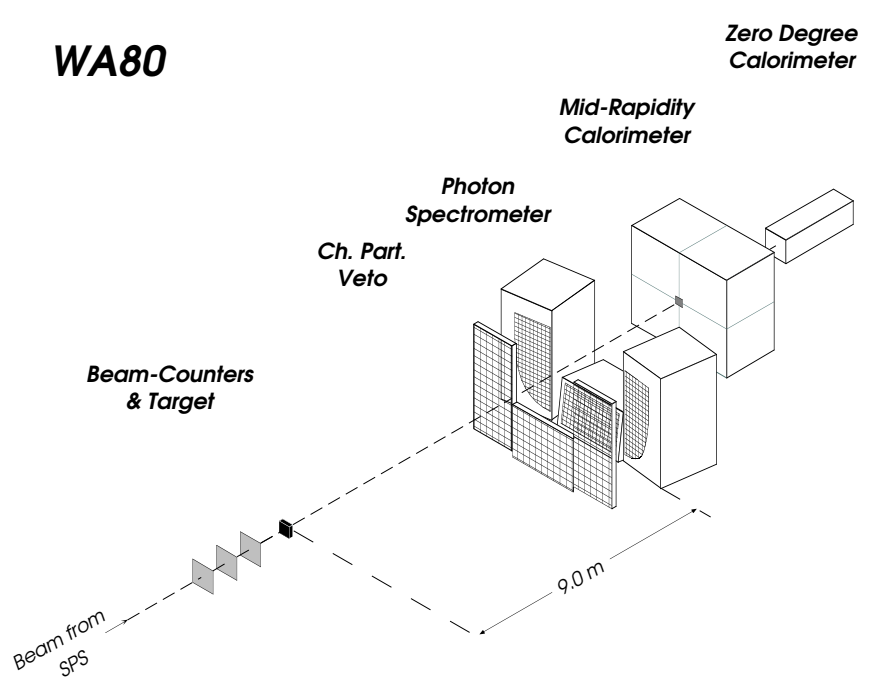

Fig. 1. WA80 experimental setup

ibrated arrays, of roughly similar size. Two arrays consisted of TF1 lead-glass of $4.0 \times 4.0 \times 40 \mathrm{~cm}^{3}\left(15 X_{0}\right)$ [19] deployed as towers to the left and right of the beam axis. The third array, located below the beam axis, was the SAPHIR lead-glass detector [20] already used in the WA80 ${ }^{16} \mathrm{O}$ run period [21] and which consisted of SF5 lead-glass modules of $3.5 \times 3.5 \times 46 \mathrm{~cm}^{3}$ $\left(18 X_{0}\right)$. The entire photon spectrometer, which was located at a distance of $9 \mathrm{~m}$ downstream of the target, provided coverage of $10 \%$ to $50 \%$ of full azimuth $\phi$ over the polar range $6.2^{\circ} \leq \vartheta_{\mathrm{lab}} \leq 13.9^{\circ}$. This resulted in a rather uniform acceptance in the rapidity range $2.1 \leq y \leq 2.9$ for reconstruction of $\pi^{0}$ and $\eta$ mesons via their $\gamma \gamma$ decay mode.

Immediately in front of the photon spectrometer was a double-layer charged particle veto (CPV) counter which covered the lead-glass region of acceptance. Each layer of the CPV consisted of streamer tubes with charge-sensitive pad readout, with pads of dimension similar to the lead-glass modules [22].

The minimum bias $(\mathrm{mb})$ trigger requires a valid signal of the beam counters and a minimum amount of transverse energy $E_{T} \gtrsim 5 \mathrm{GeV}$, detected by the Mid-Rapidity Calorimeter MIRAC [23]. Data have been taken with the $200 \mathrm{AGeV}$ sulfur beam on targets of $\mathrm{Au}\left(250 \mathrm{mg} / \mathrm{cm}^{2}\right)$ and $\mathrm{S}(205$ and $510 \mathrm{mg} / \mathrm{cm}^{2}$ ). For the present analysis 7.9 million $\mathrm{S}+\mathrm{Au}$ and 1.4 million $\mathrm{S}+\mathrm{S}$ minimum bias events were accumulated, allowing precise reconstruction of the $\pi^{0} p_{T}$ distributions over a wide range of $0.3 \leq p_{T} \leq 4.3 \mathrm{GeV} / c$ for $\mathrm{S}+\mathrm{Au}$ reactions. The minimum bias cross section has been calculated from the number of beam and minimum bias triggers and the target thicknesses, and was corrected for small contributions of background effects (typically a few percent). As a result, we obtain $\sigma_{\mathrm{m} b}=$ $1450 \mathrm{mb}$ and $3600 \mathrm{mb}$ for $\mathrm{S}+\mathrm{S}$ and $\mathrm{S}+\mathrm{Au}$ reactions, respectively. The $\mathrm{S}+\mathrm{Au}$ value is approximately $20 \%$ larger than for the 1987 WA80 data [24], due to the more biased trigger setting used then. These absolute cross sections have an overall systematic error of $10 \%$. 


\section{Pion Reconstruction and Efficiency}

For the extraction of the neutral pion yield via the $\gamma \gamma$ decay branch, all showers in the lead-glass have been considered. While additional criteria like a lateral dispersion cut [19] or the use of the CPV can improve the quality of photon identification, these cuts are of little importance for the analysis of neutral pions which are uniquely identified via their invariant mass peaks.

Hits in the detector are combined to pairs to provide distributions of pair mass vs. pair transverse momentum for all possible combinations. These distribution are obtained both for real events $\left(R\left(m_{\mathrm{in} n v}, p_{T}\right)\right)$ and for so-called mixed events $\left(M\left(m_{\mathrm{inv}}, p_{T}\right)\right)$, where a hit from one event is combined with a hit from another event with similar multiplicity. $M\left(m_{\mathrm{inv}}, p_{T}\right)$ provides a good description of the combinatorial background. It is subtracted from $R\left(m_{\mathrm{in} v}, p_{T}\right)$ to obtain the mass distribution of neutral pions.

\begin{tabular}{|c||r|r|c|r|}
\hline class & $E_{T}(\mathrm{GeV})$ & $\sigma / \sigma_{\mathrm{mb}}$ & $\begin{array}{c}\text { cell } \\
\text { occupancy }\end{array}$ & $\begin{array}{r}\text { no. of } \\
\text { events }\end{array}$ \\
\hline \hline 1 & $\leq 20.0$ & $30.0 \%$ & $0.17 \%$ & $2.37 \cdot 10^{6}$ \\
\hline 2 & $20.0-42.0$ & $20.1 \%$ & $0.43 \%$ & $942 \cdot 10^{3}$ \\
\hline 3 & $42.0-66.3$ & $15.2 \%$ & $0.82 \%$ & $461 \cdot 10^{3}$ \\
\hline 4 & $66.3-86.0$ & $9.5 \%$ & $1.24 \%$ & $539 \cdot 10^{3}$ \\
\hline 5 & $86.0-107.5$ & $10.5 \%$ & $1.55 \%$ & $1.48 \cdot 10^{6}$ \\
\hline 6 & $107.5-121.5$ & $7.0 \%$ & $1.90 \%$ & $1.02 \cdot 10^{6}$ \\
\hline 7 & $121.5-133.5$ & $4.9 \%$ & $2.10 \%$ & $717 \cdot 10^{3}$ \\
\hline 8 & $>133.5$ & $2.8 \%$ & $2.40 \%$ & $407 \cdot 10^{3}$ \\
\hline
\end{tabular}

Table 1. Centrality classes, as selected by the amount of transverse energy observed in MIRAC, and cell occupancies for ${ }^{32} \mathrm{~S}+\mathrm{Au}$ collisions.

In central reactions of ${ }^{32} \mathrm{~S}+\mathrm{Au}$ the average particle occupancy in the detector reaches values of $2.4 \%$ /module (see Table 11). This leads to a finite probability that showers in the detector overlap and influence each other. Such an overlap may result in the following modifications of the measured signals:

1. The signal of one shower is "absorbed" by another one this happens mostly to low energy showers. In such a case, the photon is lost and if it originated from a $\pi^{0}$-decay, the $\pi^{0}$ parent particle itself cannot be reconstructed and is lost.

2. The signal of one shower is influenced by that of a nearby shower. In this case, the measured energy and/or position may be changed and, thereby, the momentum of the photon may be changed. If it originated from a neutral pion, depending on how important these changes are, this may either result in

(a) changes of the apparent invariant mass such that the pion cannot be reconstructed, or

(b) changes that still allow to identify a pion by its proper invariant mass, but at a different momentum.

In addition, case $2 b$ leads to a modification of the shape of the invariant mass distribution of $\pi^{0} \mathrm{~s}$, which has to be taken into account in the extraction of the peak content.
These effects lead to a detector efficiency for pion reconstruction which depends on particle density. To study this detection efficiency, the GEANT [25] simulation package has been used to create artificial signals for the lead glass modules corresponding to neutral pions hitting the detector. In total, $\approx 10^{6} \pi^{0} \mathrm{~s}$ have been simulated with uniformly distributed transverse momenta.

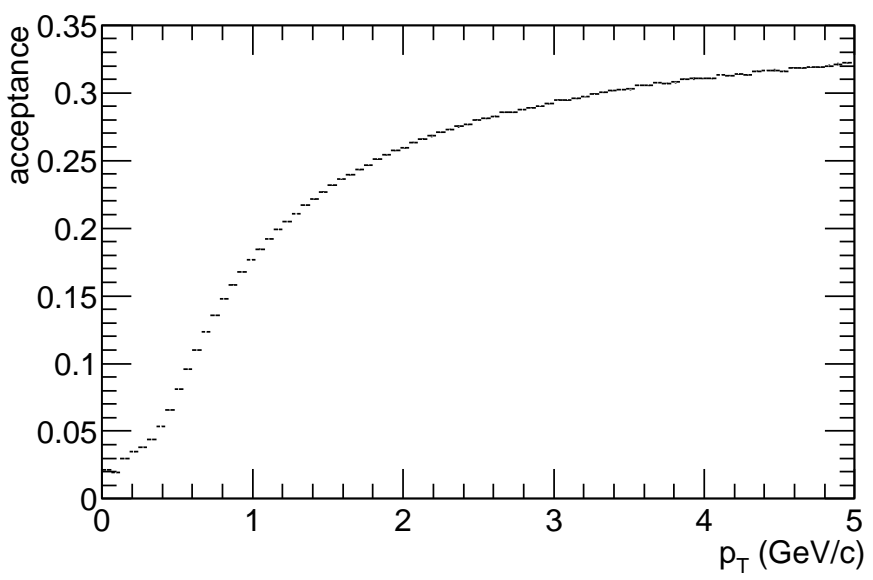

Fig. 2. Geometrical acceptance for neutral pions as a function of $p_{T}$.

The signals were then analyzed with the same analysis programs as used for real data. Analyzing them as single particles, allows to study detector effects and the analysis programs. To obtain a $\pi^{0}$ efficiency, however, these simulated photon pairs have to be studied in a realistic particle density environment. This has been done by superimposing them onto real measured events. Effects of detector noise and digitization of the photomultiplier signals are implemented. Most importantly, the simulation provides the means to extract the probability for a pion at given input transverse momentum $p_{T}^{(0)}$ to be measured with $p_{T}^{(1)}$. These probabilities measured as a function of $p_{T}^{(0)}$ can be understood as a matrix which transforms real physical distributions into measured ones. The efficiency matrix contains both effects of detection efficiency as described above, as well as the geometrical acceptance. The acceptance can be easily separated and is shown in Fig. 2 as a function of the transverse momentum. The efficiency matrix is calculated for each event class (i.e. given particle density). The representation as a matrix has the advantage that the actual shape of the momentum distributions does not need to be known beforehand.

The efficiency matrices are used to correct the measured distributions in the following way:

1. A reasonable $\pi^{0}$ distribution is chosen as initial ansatz.

2. The distribution is transformed by applying the efficiency matrix.

3. The resulting distribution $(B)$ is compared to the actual measured distribution $(A)$.

4. The input distribution is modified by multiplying it with the ratio $A / B$.

5. Steps 1-4 are iterated until $A$ and $B$ agree. 
The result of this procedure is shown in Fig. 3, where the $\pi^{0}$ reconstruction efficiency[ is plotted for the most peripheral and most central $\mathrm{S}+\mathrm{Au}$ reactions, respectively. It can be seen that for very peripheral reactions, the correction is relatively small, particularly for $p_{T} \geq 1 \mathrm{GeV} / c$. However, for the most central reactions, i.e. for the highest particle densities, the correction becomes much more important. The general behavior can be understood from the previous discussion. The drop in the $\pi^{0}$ reconstruction efficiency at low $p_{T}$ is largely caused by the increasing probability with decreasing energy to misidentify or lose a low energy photon in the radial tail of a higher energy shower, such that the (mostly low $p_{T}$ ) neutral pion cannot be reconstructed and is lost for further analysis. At large transverse momenta, the reconstruction efficiency exceeds the value 1. This is an effect of limited transverse momentum resolution on steeply falling spectra; effectively the observed distribution is broadened over the original one by the apparatus, thus giving rise to $\varepsilon_{\pi^{0}}>1$ (item $2 \mathrm{~b}$ in the list above).

This is illustrated in Fig. 1. Here, pions have been simulated with transverse momenta in a narrow range $(0.8 \mathrm{GeV} / c \leq$ $p_{T} \leq 1.0 \mathrm{GeV} / c$ ) The resulting $p_{T}$ distributions after the overlap procedure discussed above are shown as dark grey histograms. One can see that already in peripheral collisions (upper right) there is some broadening of the distribution, the effect is however much stronger in central collisions (lower right). Here a significant fraction of the pions will be measured with higher transverse momentum. For the steeply falling spectra indicated as line histograms this may result in a larger apparent number of pions in the higher $p_{T}$ bins and thus lead to an "efficiency" $>1$. An equivalent broadening effect can be seen in the experimental data e.g. in the invariant mass spectra displayed on the left hand side of Fig. A. Overlap effects lead to an additional high mass tail of the pion peak in central collisions.

The systematic errors on the measured transverse momentum spectra are dominated by the following contributions:

\footnotetext{
1 We refer now to the "pure" efficiency without any effect from the geometrical acceptance.

2 The estimates given here apply to central collisions where the errors are largest.
}

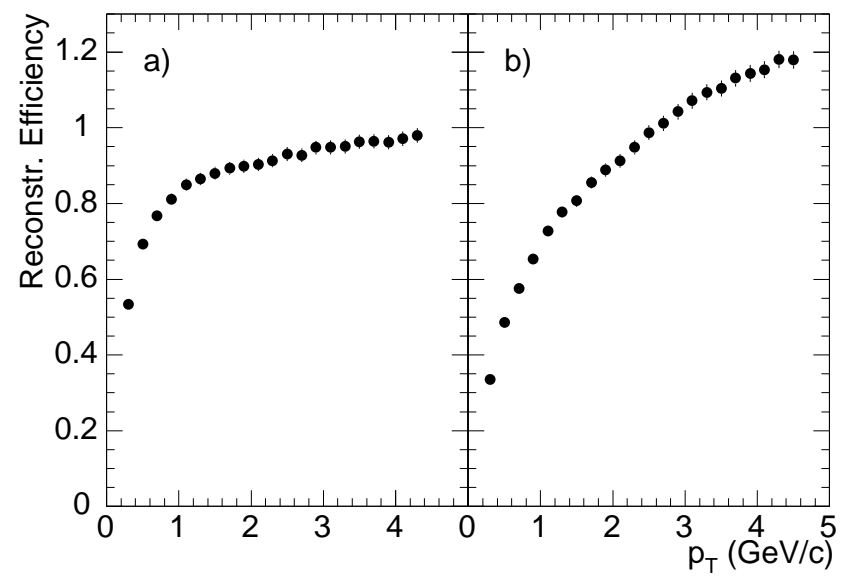

Fig. 3. Neutral pion reconstruction efficiency for very peripheral (a) and very central ${ }^{32} \mathrm{~S}+\mathrm{Au}$ reactions (b).
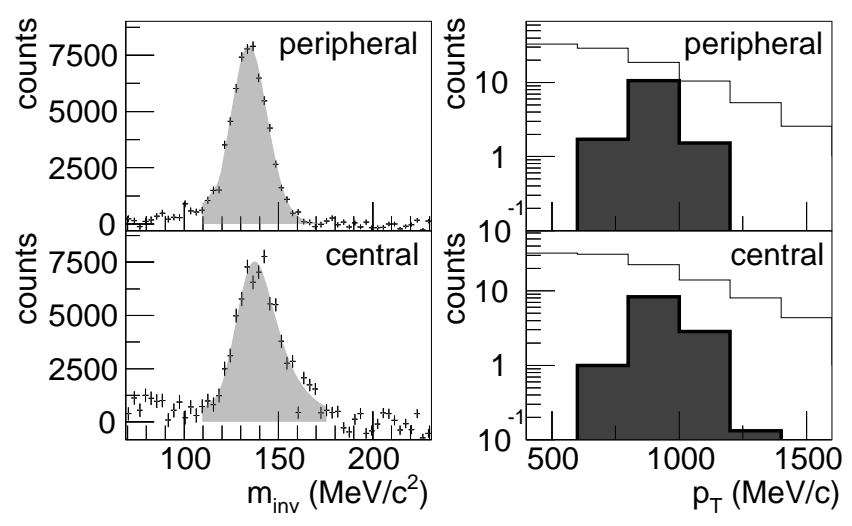

Fig. 4. Influence of shower overlap on the neutral pions with $0.8 \mathrm{GeV} / c \leq p_{T} \leq 1.0 \mathrm{GeV} / c$ for peripheral and central collisions. The plots on the left hand side show two-photon invariant mass spectra, where the light grey area indicates the integration range for the pion extraction. The dark grey histograms on the right hand side show the $p_{T}$ distribution of pions simulated with $0.8 \mathrm{GeV} / c \leq p_{T} \leq$ $1.0 \mathrm{GeV} / c$ after overlap effects from other showers are included. For reference, the relevant region of the $p_{T}$-spectra is also shown. See text for further discussion.

- An uncertainty in the absolute calibration of the momentum scale of $1 \%$. This may be translated into an uncertainty in the yields of a few $\%$ at low $p_{T}$ rising to $\approx 13 \%$ at $p_{T}=$ $3.5 \mathrm{GeV} / c$.

- Uncertainties in the $\pi^{0}$ extraction which include the error in the determination of the invariant mass peak content and the propagation of the energy resolution through the acceptance and efficiency corrections. This leads to an error of $16 \%$ at $p_{T}=0.5 \mathrm{GeV} / c$ which decreases to below $3 \%$ at high $p_{T}$.

These two major contributions to the systematic errors and their quadratic sum are shown in Fig.5.

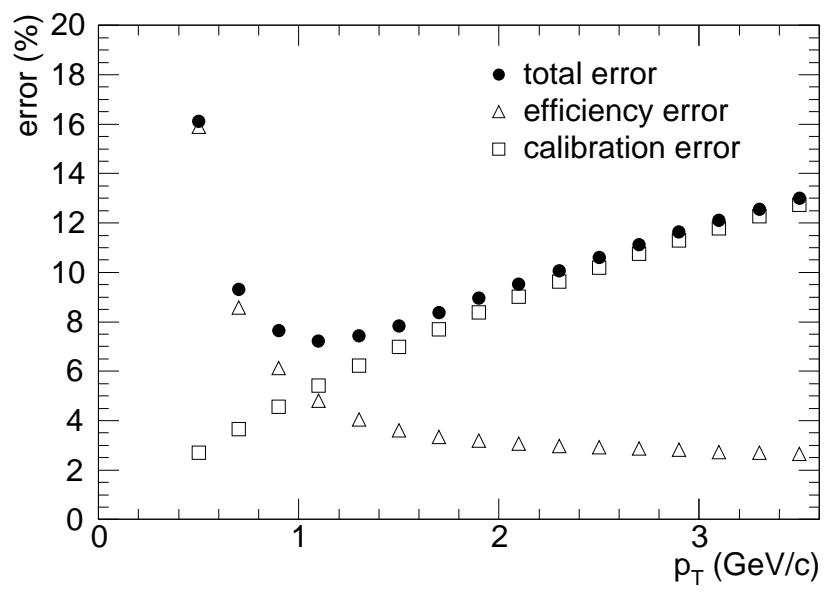

Fig. 5. Systematic errors of the extraction of the $\pi^{0}$ yields for central $\mathrm{S}+\mathrm{Au}$ collisions as a function of $p_{T}$.

\footnotetext{
3 This error contribution is much smaller in the ratios of photon or pion spectra as e.g. used for the direct photon analysis [18].
} 


\section{Results}

\subsection{Nuclear Reactions}

Efficiency corrected $\pi^{0}$ invariant cross sections measured in the rapidity range $2.1 \leq y \leq 2.9$ are presented in Fig. 6 for $\mathrm{S}+\mathrm{S}$ and $\mathrm{S}+\mathrm{Au}$ reactions. The data are shown for central, peripheral and minimum bias trigger conditions. The central event class of $7.7 \%$ of $\sigma_{\mathrm{m} b}$ in $\mathrm{S}+\mathrm{Au}$ collisions corresponds to complete overlap of the $\mathrm{S}$ nucleus with the Au target, with an average of $\approx 110$ participating nucleons, to be compared to an average of $\approx 11$ participating nucleons for the $30 \%$ most peripheral events. From a first inspection it is seen that the slopes appear systematically flatter for central collisions than for peripheral ones. This might indicate a higher temperature in more violent central collisions. Although the slope parameters cannot be identified a priori with a temperature in a thermodynamic sense, we shall often use the label "temperature" in the following. I Superimposed on the data are exponentials $d \sigma / d m_{T}^{2} \propto \exp \left(-m_{T} / T\right)$ fitted to the region $0.8 \mathrm{GeV} / c^{2}$ $\leq m_{T} \leq 2.0 \mathrm{GeV} / c^{2}$. The fitted slope parameters are indicated. In this parameterization of the intermediate $m_{T}$ data, the flattening of the distributions with increasing centrality results in a higher apparent temperature. The high precision and the large $m_{T}$ range of the present $\pi^{0}$ data allows to recognize, however, that the spectral shapes are only very poorly described by an exponential curve. Instead, a concave behavior reminiscent of pp data at similar or higher $\sqrt{s}$ is observed over the whole $m_{T}$ range. Its properties are more systematically analyzed by calculating the local slope, defined as

$$
T_{\mathrm{loc}}^{-1}=-\frac{d}{d m_{T}}\left[\log \left(E \frac{d^{3} \sigma}{d p^{3}}\right)\right] .
$$

The results are plotted in Fig. 7f for the $\mathrm{S}+$ Au data. Here, each individual value of $T_{l o c}$ has been determined locally from three adjacent data points of Fig. 6. Such an analysis puts very high demands on the statistical and systematic uncertainties in order to keep point-to-point fluctuations at an acceptable level. Shown together with the heavy ion data are pp minimum bias data which will be discussed in section 4.2.

As expected, the observed local slopes are not constant, as would be the case for a purely exponential distribution, but change continuously over the measured $m_{T}$ range both for peripheral and central collisions. This again indicates that fits with one or two exponentials, which have often been presented elsewhere (e.g. Ref. [26]), can only provide a crude approximation valid over a restricted $m_{T}$ range. Fig. 77 also demonstrates that the slope parameters in the intermediate $m_{T}$ range are systematically higher for central compared to peripheral data.

In order to systematically compare data from different systems and/or taken under different trigger conditions it is often more convenient to apply a description in terms of a functional form. According to the above discussion, such a parameterization must allow for $p_{T}$ dependent variations in the curvature of the spectra. At high $p_{T}$, where perturbative QCD becomes applicable, the spectra are expected to attain a power-law behavior, as observed in numerous high energy pp measurements (see

\footnotetext{
${ }^{4}$ All slope parameters will be given in units $\mathrm{MeV}$.
}

for example Ref. [27]). The heavy-ion data of this experiment seem to follow that trend even into the intermediate $p_{T}$ range. Therefore, a parameterization originally inspired by QCD [28] and successfully applied already to pp data [27] has been chosen to fit to the spectra:

$$
E \frac{d^{3} \sigma}{d p^{3}}=C\left(\frac{p_{0}}{p_{T}+p_{0}}\right)^{n}
$$

with $C, p_{0}$ and $n$ taken as free parameters. A link to the more familiar exponential slope parameter $T$ is obtained from the derivative of Eq. 2 according to

$$
T_{\text {power-law }}=-\frac{f\left(p_{T}\right)}{\frac{\partial f\left(p_{T}\right)}{\partial p_{T}}}=\frac{p_{0}}{n}+\frac{1}{n} \cdot p_{T} .
$$

Thus, $p_{0} / n$ characterizes the slope of the transverse momentum spectra in the limit $p_{T} \rightarrow 0$, while $1 / n$ characterizes its gradient along $p_{T}$, i.e. the strength of the concave curvature. The analytical form of Eq. 3 can in that way be compared directly to the numerical values of the local slopes presented in Fig. [7. As stressed by Hagedorn [28], and immediately recognized from Eq. 3. $p_{0}$ and $n$ are highly correlated. We have therefore chosen to express Eq. 2 as a function of the local slopes $T_{1}$ and $T_{2}$ at two fixed values of $p_{T}(1 \mathrm{GeV} / c$ and $3 \mathrm{GeV} / c)-$ these are less correlated and therefore better suited as fit parameters.

Fits to the data using Eq. 2. 2 are presented in Fig. 8. The extracted parameters are compiled in Table together with the results of analogous fits to $p+p$ data [2]. All slope parameters are observed to increase smoothly with the centrality of the reaction, e.g. $T_{1}$ increases from approximately $196 \mathrm{MeV}$ in peripheral collisions to approximately $206 \mathrm{MeV}$ in central collisions.

Another way to investigate the differences between peripheral and central data in a model independent way is to study the ratio of the transverse momentum distributions as a function of

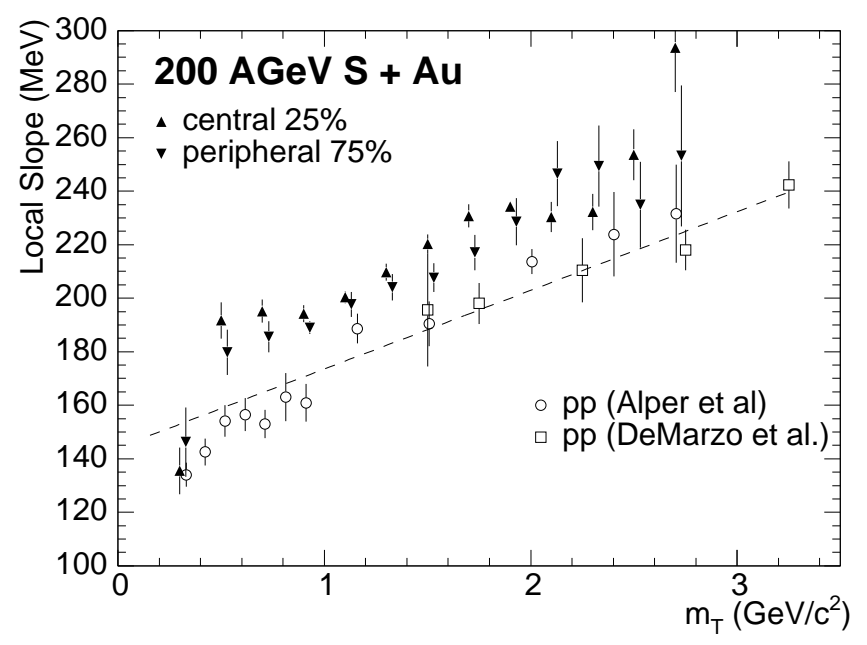

Fig. 7. Local slope presentation of central and peripheral $\mathrm{S}+\mathrm{Au} \pi^{0}$ data drawn together with minimum bias pp data [2]. The dashed line shows the local slopes calculated according to Eq. 3 from the fit of Eq.2 to the pp data. 


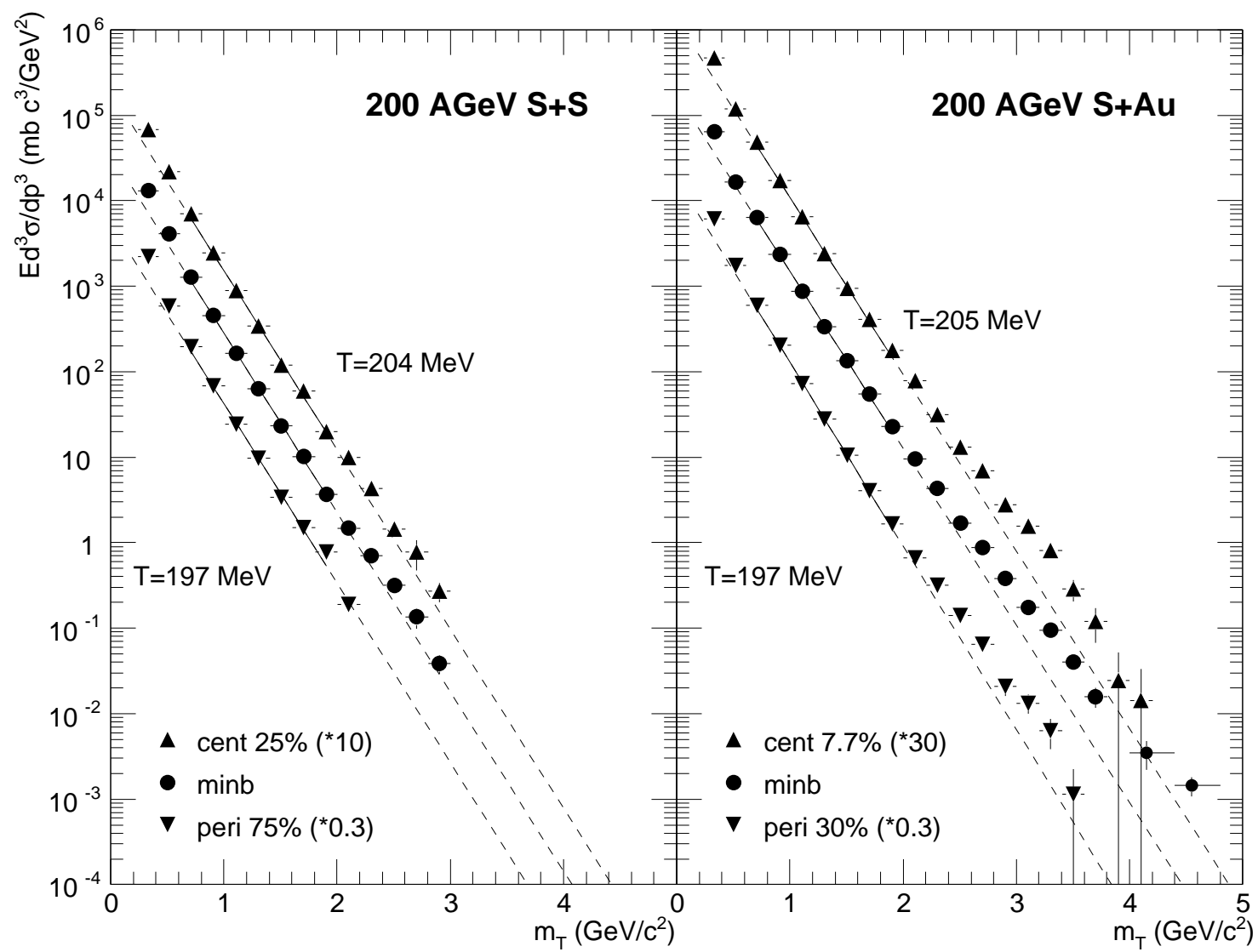

Fig. 6. Invariant cross sections of $\pi^{0}$ mesons from reactions of $\mathrm{S}+\mathrm{S}$ (left) and $\mathrm{S}+\mathrm{Au}$ (right) at $200 A \cdot \mathrm{GeV}$ measured in the rapidity range $2.1 \leq y \leq 2.9$. The events are selected for centrality with the percentage of the minimum bias cross section as indicated. The lines drawn with the data are exponentials fitted to the region $0.8 \mathrm{GeV} / c^{2} \leq m_{T} \leq 2.0 \mathrm{GeV} / c^{2}$ with slope parameters as indicated.

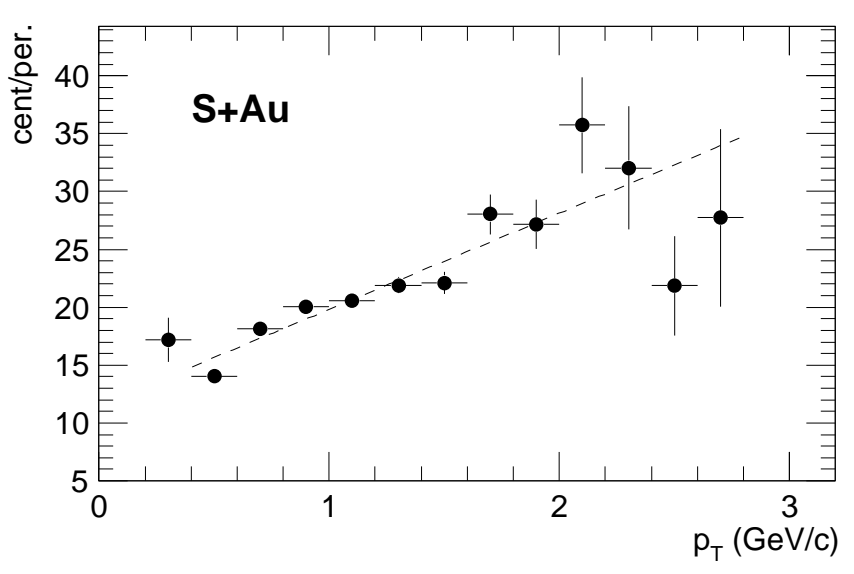

Fig. 9. Ratio of $\pi^{0}$ multiplicities from central (7.7\%) and peripheral $(30 \%) \mathrm{S}+\mathrm{Au}$ reactions as a function of $p_{T}$.

$p_{T}$. Fig. 9 shows that with increasing $p_{T}$, the pion multiplicity in central data is enhanced relative to that of peripheral data. Measured over the dynamic range of the $\mathrm{S}+\mathrm{Au}$ data, a variation by a factor of $\gtrsim 2$ is observed. Similar findings in the cross section ratios have been reported earlier by us for central and peripheral $\mathrm{O}+\mathrm{Au}$ reactions at $200 \mathrm{GeV}$ [21].

The mean transverse momentum $\left\langle p_{T}\right\rangle$ is an observable containing important information about the reaction dynamics of the system and is often used to provide a convenient means of comparison among different experiments and model calculations. The evaluation of $\left\langle p_{T}\right\rangle$ is usually achieved analytically by using parameterizations of the experimental distributions (see below). Unless the cross section is measured down to very low $p_{T}$ and the functional form describes the data well, this method may lead to systematic errors due to the dominance of the low $p_{T}$ cross section. If only the variation of $\left\langle p_{T}\right\rangle$ as a function of another observable, like for example the centrality, is of importance, it is more advantageous to calculate instead the truncated mean transverse momentum above a certain threshold directly from the experimental data. This can be done by the relation:

$$
\left\langle p_{T}\right\rangle_{d}=\left(\int_{d}^{\infty} p_{T} \frac{d N}{d p_{T}} d p_{T} / \int_{d}^{\infty} \frac{d N}{d p_{T}} d p_{T}\right)-d
$$

Here, $d=0.4 \mathrm{GeV} / c$ is chosen according to the lowest $p_{T}$ data point where systematic uncertainties imposed by acceptance and efficiency corrections are still well under control. For a purely exponential invariant cross section, i.e. $d \sigma / d p_{T}^{2} \propto$ 


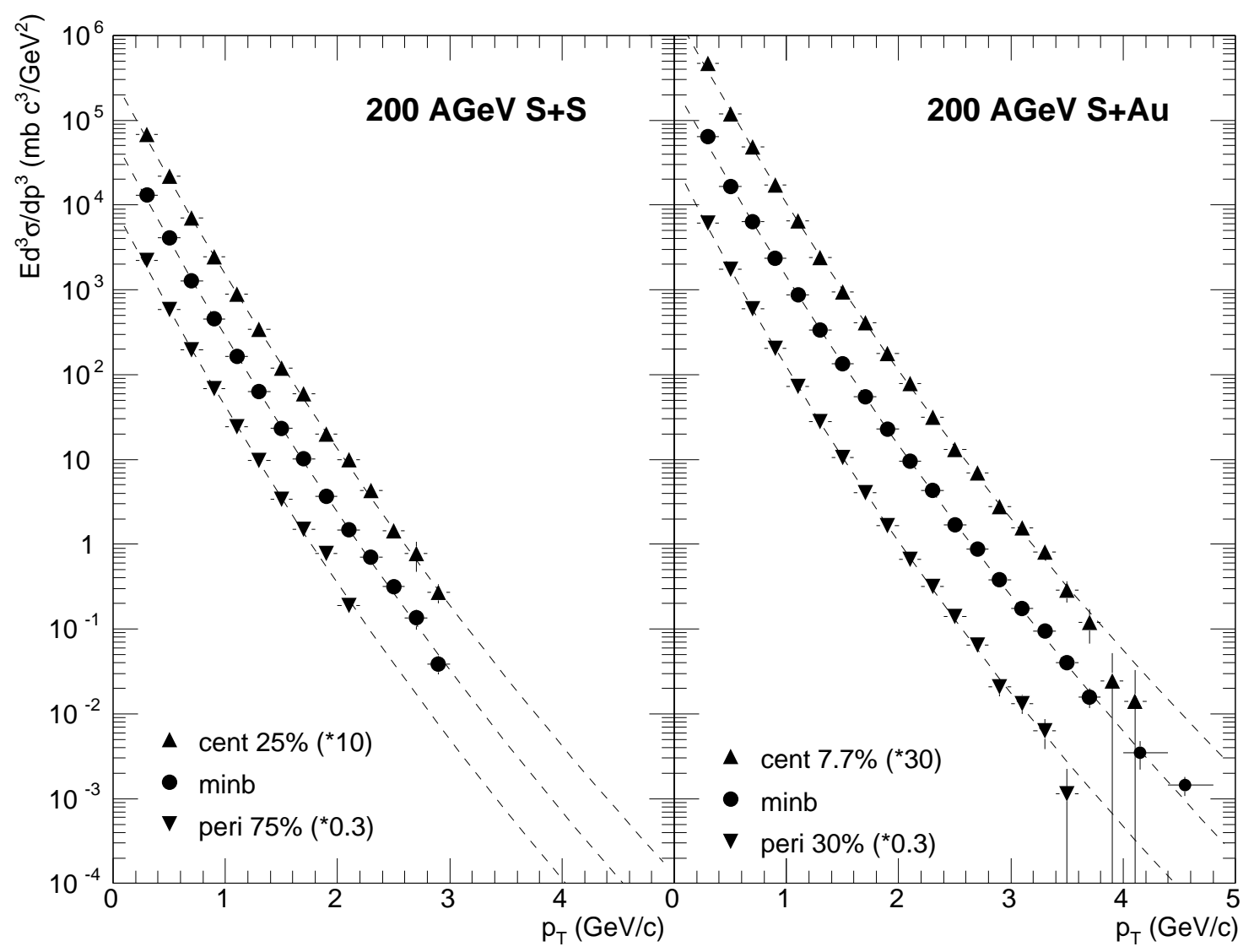

Fig. 8. Fits to $200 A \cdot \mathrm{GeV} \mathrm{S}+\mathrm{S}$ and $\mathrm{S}+\mathrm{Au}$ data using Eq. 2 with parameters given in Table日.

$\exp \left(-p_{T} / T\right)$, and for $d=0$, one immediately finds $\left\langle p_{T}\right\rangle_{d=0}=$ $2 T$. For finite values of $d$, however, this simple relation does not hold and $\left\langle p_{T}\right\rangle_{d}$ decreases as a function of $d$.

Most interesting in connection with the thermodynamical picture of the compressed and excited heavy ion system is the variation of $\left\langle p_{T}\right\rangle$ with $E_{T}$ or the multiplicity $d N_{c h} / d \eta$ of produced particles. We therefore have studied $\left\langle p_{T}\right\rangle_{d}$ of identified $\pi^{0}$ mesons as a function of the transverse energy. It has been demonstrated earlier [24] that $E_{T}$ is proportional to the total number of participating nucleons $N_{\text {part }}$. For $\mathrm{S}+\mathrm{Au}$ reactions $N_{\text {part }}$ has been calculated for the different centrality cuts by Glauber calculations. In Fig. 10 the values of $\left\langle p_{T}\right\rangle_{d}$ as a function of $N_{\text {part }}$ are shown. A rise in $\left\langle p_{T}\right\rangle_{d}$ with increasing $N_{\text {part }}$ is observed, which again reflects the increase with centrality of the slope parameters of the exponential fits in Fig. 6, or of the local slopes shown in Fig. 7. The values seem to saturate at $\approx 290 \mathrm{MeV} / \mathrm{c}$ for medium-central to central collisions. These relatively small values may be compared with those obtained from a complete integration of the fits with Eq.2. For central $\mathrm{S}+\mathrm{Au}$ collisions one obtains $\left\langle p_{T}\right\rangle \approx 380 \mathrm{MeV} / \mathrm{c}$ - these values, however, have a large uncertainty due to the low $p_{T}$ extrapolation.

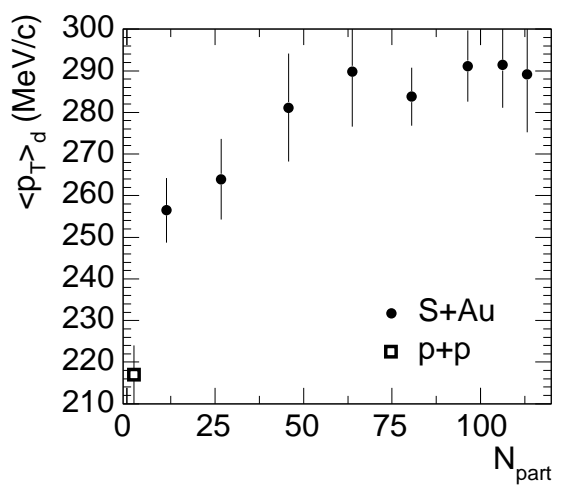

Fig. 10. Truncated mean transverse momentum $\left\langle p_{T}\right\rangle_{d}$ of $\pi^{0}$ mesons as defined by Eq. 4 plotted as a function of the average number of participants $N_{\text {part }}$. The data points correspond to the $8 E_{T}$ based centrality selections listed in Table 11. The open square shows $\left\langle p_{T}\right\rangle_{d}$ for $\mathrm{pp}$ collisions extracted from the fit with Eq. 2. A cut parameter $d=0.4 \mathrm{GeV} / c$ was used.

\subsection{Comparison to $p p$ and $p A$ data}

The transverse momentum spectra presented for $\mathrm{S}+\mathrm{Au}$ reactions unambiguously demonstrate distinct variations as a function of event centrality. In order to learn, how these characteristic changes can be attributed to differences in the size of the 
Table 2. Parameters obtained by fitting Eq. 2 to the invariant cross section of neutral pions from different reaction systems and for different classes of centralities. The parameter $T_{1}$ and $T_{2}$ are local slopes according to Eq 3 . The pp data taken at $\sqrt{s}=23.5 \mathrm{GeV}$ have been scaled to $\sqrt{s}=19.4 \mathrm{GeV}$ before fitting according to the recipe given in [29].

\begin{tabular}{|c|c|c|c|c|c|c|c|c|}
\hline & & $\begin{array}{c}\text { Fraction } \\
\text { of } \sigma_{\mathrm{m} b}\end{array}$ & $\begin{array}{c}p_{0} \\
(\mathrm{GeV} / c)\end{array}$ & $n$ & $\begin{array}{c}p_{0} / n \\
(\mathrm{MeV} / c)\end{array}$ & $\begin{array}{c}T_{1} \text { at } 1 \mathrm{GeV} \\
(\mathrm{MeV})\end{array}$ & $\begin{array}{c}T_{2} \text { at } 3 \mathrm{GeV} \\
(\mathrm{MeV})\end{array}$ & $\chi_{\text {red }}^{2}$ \\
\hline \multirow{5}{*}{$\mathrm{S}+\mathrm{Au}$} & centr. & $7.7 \%$ & $5.6 \pm 0.5$ & $32.0 \pm 2.0$ & $174.5 \pm 2.6$ & $205.7 \pm 1.0$ & $268.1 \pm 3.2$ & 2.2 \\
\cline { 2 - 10 } & centr. & $25 \%$ & $5.7 \pm 0.3$ & $32.8 \pm 1.2$ & $174.3 \pm 1.5$ & $204.7 \pm 0.5$ & $265.7 \pm 1.9$ & 3.2 \\
\cline { 2 - 10 } & & $100 \%$ & $6.5 \pm 0.3$ & $36.4 \pm 1.4$ & $178.6 \pm 1.4$ & $206.1 \pm 1.8$ & $261.0 \pm 1.8$ & 2.2 \\
\cline { 2 - 10 } & per. & $75 \%$ & $5.3 \pm 0.4$ & $31.6 \pm 2.0$ & $166.7 \pm 2.4$ & $198.3 \pm 0.7$ & $261.5 \pm 3.5$ & 0.6 \\
\hline \multirow{3}{*}{$\mathrm{S}+\mathrm{S}$} & per. & $30 \%$ & $4.9 \pm 0.5$ & $30.2 \pm 2.3$ & $163.0 \pm 2.8$ & $196.1 \pm 0.8$ & $262.3 \pm 4.3$ & 0.6 \\
\cline { 2 - 10 } & & $100 \%$ & $5.6 \pm 1.4$ & $33.5 \pm 6.6$ & $166.8 \pm 6.5$ & $196.6 \pm 8.7$ & $256.4 \pm 8.7$ & 0.7 \\
\hline \multirow{2}{*}{$\mathrm{p}+\mathrm{p}$} & pentr. & $75 \%$ & $6.2 \pm 4.1$ & $36.2 \pm 20.5$ & $170.2 \pm 11.5$ & $197.9 \pm 2.6$ & $253.2 \pm 19.2$ & 1.2 \\
\hline
\end{tabular}

interacting system, it is of interest to compare with data from $\mathrm{pp}$ and $\mathrm{pA}$ collisions in a consistent way. Unfortunately, a rigorous comparison cannot be made free of assumptions, since the relevant data from different experiments, in general, do not correspond to the same $\sqrt{s}$ and rapidity range. The large body of experimental data collected for $\mathrm{pp}$ reactions, however, may be used to extract appropriate scaling laws of transverse momentum spectra as a function of $\sqrt{s}$ and rapidity $y$ and thus allow to adopt these data to the corresponding values of our experiment. In this work, we have employed the empirical concept which is presented in Ref. [31] together with data from Refs. [2, 3, 4, 29, 32, 30]. The minimum bias pp data of Ref. [2] 30], taken at $\sqrt{s}=23.5 \mathrm{GeV}$ and at slightly more forward rapidities, were thus scaled appropriately to the conditions of the WA80 experiment. The systematic uncertainty introduced by this procedure is included in the error bars shown.

A first comparison of the $\pi^{0}$ spectra from pp collisions with that of S-induced reactions was shown in Fig. 7. The slope parameters $T_{\text {loc }}$ smoothly grow from approx. $150 \mathrm{MeV}$ at $m_{T} \approx$ $0.3 \mathrm{GeV} / c^{2}$ to almost $250 \mathrm{MeV}$ at $m_{T} \gtrsim 3.0 \mathrm{GeV} / c^{2}$, demonstrating convincingly the strong concave shape of the transverse momentum distributions. The local slopes both in $p+p$ and heavy ion data increase in a similar way when going from low $p_{T}$ to high $p_{T}$, thus indicating that the curvature is very similar in both cases. The main difference is in the systematically higher values of the local slopes in heavy ion reactions. The slopes of the $p_{T}$ spectra in $\mathrm{pp}$ and nucleus-nucleus collisions differ over the whole $p_{T}$ range which indicates the occurence of strong nuclear effects in particle production.

The variations seen in the local slopes are reflected in the truncated mean transverse momentum (Fig. 10). Here, $\left\langle p_{T}\right\rangle_{d}$ has also been calculated for $p p$ data from a parameterization. The value of $217 \mathrm{MeV} / \mathrm{c}$ is considerably below those for the

\footnotetext{
5 As discussed above, small differences in the curvature can be seen from the fits of Eq. 2
}

$\mathrm{S}+\mathrm{Au}$ data, even for the most peripheral collisions. Apparently, the number of participating nucleons even in the peripheral collisions $(\approx 11)$ is too large to treat them as identical to pp collisions.

The ratio of multiplicities is shown in Fig. 11 as a function of $p_{T}$ for the same set of data. In this presentation, we find an increase of the $(\mathrm{S}+\mathrm{Au})_{\text {cent }} / \mathrm{pp}$ ratio with $p_{T}$ by almost a factor of 10 over the dynamic range of the experiment and for $(\mathrm{S}+\mathrm{Au})_{\text {per }} / \mathrm{pp}$ an increase by a factor of $\approx 4$. We would like to point out that this large increase of the cross section ratio at a given $p_{T}$ may be seen as a consequence of the differences of the local slope at lower values of $p_{T}$ (see Fig. 7). The "high $p_{T}$ enhancement" of the cross sections therefore appears not to be
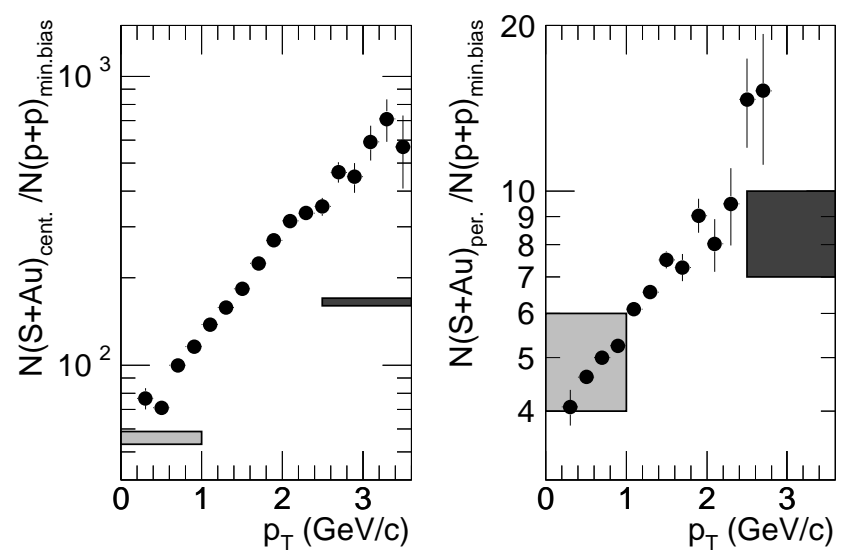

Fig. 11. Ratio of central (left) and peripheral (right) $200 A \cdot \mathrm{GeV}$ $\mathrm{S}+\mathrm{Au}$ to $\mathrm{p}+\mathrm{p}$ minimum bias $\pi^{0}$ multiplicities as a function of $p_{T}$. The pp data are scaled to the $\sqrt{s}$ of the present data. Indicated as shaded areas are the ratio of the number of participants at low $p_{T}$ and the ratio of the number of binary collisions at high $p_{T}$. 


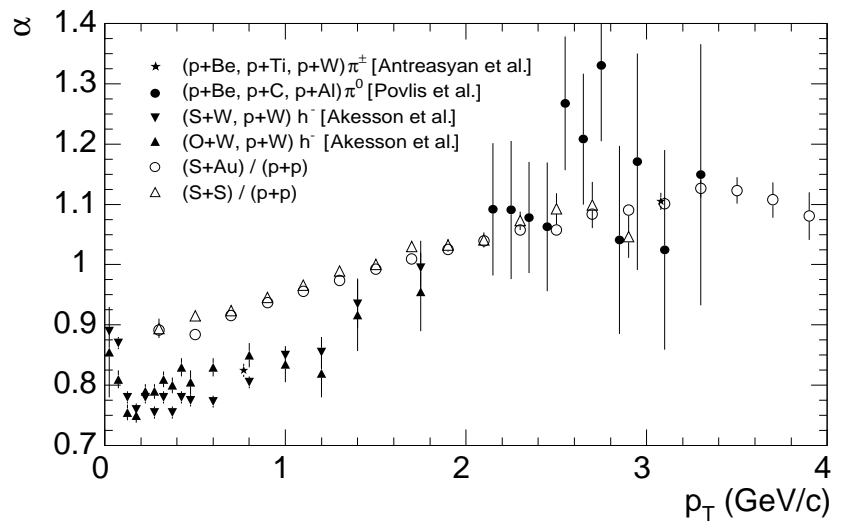

Fig. 12. Transverse momentum dependence of $\alpha\left(p_{T}\right)$ from expression 5 for the present $\pi^{0}$ data from minimum bias $\mathrm{S}+\mathrm{S}$ and $\mathrm{S}+\mathrm{Au}$ at $200 A \cdot \mathrm{GeV}$ compared with data from other reaction systems [32, 33, 34 .

an independent feature at high $p_{T}$ but may rather be considered as the result of the different slopes over the complete $p_{T}$ range.

For comparison Fig. 11 includes estimates of the ratios of the number of participants (light grey area at low $p_{T}$ ) and of the number of binary collisions (dark grey area at high $p_{T}$ ). The pion production seems to scale approximately with the number of participants at low $p_{T}$, but increases much more strongly at high $p_{T}$ in central $\mathrm{S}+\mathrm{Au}$ collisions compared to $\mathrm{pp}$ than expected from the number of binary collisions.

A similar variation of the cross section ratios was already observed by Cronin et al. [7] in a comparison of $\mathrm{p}+\mathrm{p}$ and $\mathrm{p}+\mathrm{A}$ data. The systematic behavior could be successfully parameterized by the phenomenological expression

$$
E \frac{d^{3} \sigma}{d p^{3}}(\mathrm{p}+\mathrm{A})=A^{\alpha\left(p_{T}\right)} E \frac{d^{3} \sigma}{d p^{3}}(\mathrm{p}+\mathrm{p})
$$

where, apart from the expected $\sqrt{s}$ dependence, the parameter $\alpha\left(p_{T}\right)$ was found to depend only weakly on rapidity [35] but strongly on $p_{T}$. At lower $p_{T}, \alpha$ turned out to be approximately 0.7 which in Eq. 5 gives a factor close to $A^{2 / 3}$ as expected for an opaque target nucleus. For higher transverse momenta, $\alpha$ was found to increase with $p_{T}$ indicating a growing participation of the nuclear volume, approaching $\alpha=1$ in the limit of full participation of the target nucleus. The surprising observation of $\alpha>1$ for the highest $p_{T}$ values, called 'anomalous nuclear enhancement', was subsequently interpreted as an effect of multiple scattering of the incident partons [9].

For $A+B$ collisions (where $A$ and $B$ denote heavy nuclei), the straightforward extension of Eq.5 writes

$$
E \frac{d^{3} \sigma}{d p^{3}}(\mathrm{~A}+\mathrm{B})=(A \cdot B)^{\alpha\left(p_{T}\right)} E \frac{d^{3} \sigma}{d p^{3}}(\mathrm{p}+\mathrm{p})
$$

and has successfully been applied to experimental data in Ref. [34]. Fig. 12] shows a compilation of $\alpha\left(p_{T}\right)$ obtained from the present data together with other data sets. The investigated projectiles, targets, and detected particle species are indicated together with their references. One should note that the statistical accuracy of the present data even at high $p_{T}$ is comparable to the data of [32] and is superior to that of the other data sets.
It appears that Eq. 6 provides an almost universal parameterization for all systems with $\alpha>1$ at high $p_{T}$. The differences of the exponent of the present data compared to NA34 at low $p_{T}$ [34 may be caused by the more backward rapidity region of the NA34 experiment. However, the data for $\mathrm{S}+\mathrm{S}$ and $\mathrm{S}+\mathrm{Au}$ data do also disagree with the data of [32] at $p_{T}=$ $0.77 \mathrm{GeV} / c$ which are measured in a similar rapidity region. It should be noted that the different trigger biases are another source of systematic errors in such a comparison of minimum bias cross sections. It may also be relevant that the present data are compared to a compilation of pp data, while in [32] one particular data set measured within the same experiment has been used. Different normalizations of the pp data could therefore contribute to a difference in $\alpha$.

\section{Discussion}

The slope and the curvature of the transverse momentum spectra, studied as a function of projectile energy, rapidity, and multiplicity, carry important information about the reaction dynamics. All spectra of produced particles emerging from $\mathrm{pp}, \mathrm{p} A$, and $A B$ collisions are known to exhibit in a reasonable approximation an exponential shape which might be regarded as indication for a thermal emission mechanism. In fact, for a thermalized system the particle $p_{T}$ spectra reflect the temperature of the system at the time of decoupling. This idealized picture is altered, if there is collective motion in the system, such as hydrodynamic flow. In such a case, the transverse velocities of particles are to be considered a sum of a random thermal component and an ordered collective expansion velocity. If one or both components change between different reaction systems or impact parameter regions, the transverse momentum distributions are expected to be affected. The picture of thermal emission has, despite its simplicity, proven very successful. The assumptions made in that interpretation might, however, be questioned particularly in pp systems because of the small volume available for particle production. Surprisingly, the same concept has recently even been applied to hadron production in $e^{+} e^{-}$collisions at LEP energies and was able to reproduce many of the observed particle ratios [36]. One may take the success of these models as a hint for the validity of statistical concepts, which however do not necessarily imply a thermal origin.

With the growing amount of data and their improved precision it has been well established that $p_{T}$-spectra of all systems, when measured with sufficient accuracy and over a large $p_{T}$ range, display a curved structure which cannot be fit by a simple exponential. Therefore, fits often have been made with either a single exponential in a restricted $p_{T}$ region and deviations have then been termed "low $p_{T}$ enhancement" or "high $p_{T}$ enhancement" respectively, or a superposition of two or more exponentials has been applied over a larger range of $p_{T}$. Without a physical picture, the former approach is rather misleading since it arbitrarily defines a "normal" behavior and deviations from that, and the latter one easily leads to an inflation of fit parameters.

On the other hand, there are in fact indications for different mechanisms at high and low $p_{T}$ which have to be treated separately and should be included appropriately in a complete 
description of the full $p_{T}$ spectrum. For $p_{T} \gtrsim 2 \mathrm{GeV} / c$ spectra from pp collisions have successfully been described by perturbative QCD calculations [27]. The "nuclear effects" observed in $\mathrm{p} A$ reactions have then been discussed in terms of multiple parton scattering. A generalization and extension of these calculations for $\mathrm{AB}$ collisions is provided by the kinetic parton cascade model [37,38], which treats the reaction - for processes of large momentum transfer - on the basis of interacting quarks and gluons. Since this model employs perturbative QCD, it can only be applied to large $p_{T}$ processes. For the much more abundant low $p_{T}$ processes, presently only phenomenological models can be applied.

Several codes are available, which are based on phenomenological string models [10, 11, 12] and use experimental data from elementary collision processes as input. Within these models, after appropriately accounting for the nuclear geometry, a heavy ion collision is treated as a superposition of individual nucleonnucleon collisions. Obviously, such an approach neglects any collective effect which might be present in an extended strongly interacting system. Comparison with various data shows that such a straightforward extrapolation from pp collisions provides a reasonable description, particularly for the intermediate $p_{T}$ range. This indicates that minimum bias heavy ion collisions in this region do not exhibit dramatic effects extending beyond the straightforward extrapolation from pp collisions. Deviations from standard string model simulations are observed particularly for central nuclear collisions both at low and high $p_{T}$. Various attempts have thus been made to improve the description of the data by including additional mechanisms into the string models. These extensions include hard scattering processes [11], rescattering of the produced particles in the target nucleus and among themselves [12], production of quark clusters [39], or color rope formation [40].

As an example, VENUS 4.12 [12] and FRITIOF 7.02 [11] calculations are shown in Fig. 13 together with the $\pi^{0}$ spectrum from central $\mathrm{S}+\mathrm{Au}$ collisions. The simulated events have been filtered with the experimental acceptance and no normalization to the cross section of the data is applied. The description of the spectra is reasonably good at $p_{T} \leq 1 \mathrm{GeV} / c$. At higher $p_{T}$, FRITIOF falls below the data and is about a factor of 5 too low at $p_{T} \geq 2 \mathrm{GeV} / c$. The agreement between data and VENUS is better, which may be attributed to the rescattering of produced particles implemented in this model. VENUS however also starts to deviate at higher $p_{T}$; it is more than a factor of 2 too high at $p_{T} \geq 3 \mathrm{GeV} / c$. If rescattering in the nuclear medium is included, the description at high $p_{T}$ is improved somewhat, at the expense, however, of additional parameters in the model. Still the description is not perfect.

A completely different approach starts from nuclear hydrodynamics and treats the heavy ion collision as an expanding thermodynamical system characterized by a temperature, chemical potential, and expansion velocity $[13,14,41,42]$. Especially for central collisions of heavy nuclei thermal equilibrium seems plausible, at least locally, due to the frequent rescattering of produced particles in the extended medium. This picture is supported by the large rapidity shifts of produced particles observed in central compared to peripheral collisions [24,43. The extraction of freeze-out temperatures from measured pion $p_{T}$ spectra within this model is complicated for sev- eral practical and conceptual reasons. First, it has been recognized that a significant fraction of the observed pions originate from decays of short-lived heavier resonances. This leads to characteristic deviations from a single exponential and, particularly at low $p_{T}$, to a more curved spectrum, as observed experimentally [44]45]. By including all contributing resonances, the authors of [46] were able to describe the experimental $p_{T}$ spectra of pions from pp systems. The success of this approach, however, does not necessarily prove that thermal equilibrium is in fact reached in such systems.

The same procedure has been applied to heavy ion systems using a computer program provided by the authors of [42]. Here, however, in addition to the most important resonance decays, transverse flow has also been included in the calculations. This leads to a "blue shift" of the apparent temperatures relative to the freeze-out temperatures of the system. By fixing the baryonic chemical potential to $\mu_{B}=200 \mathrm{MeV}$ and varying the temperature and expansion velocity, good fits to the experimental data have been obtained. An example of such a calculation applied to the present data is shown in Fig. 14.

While in earlier analyses of $p_{T}$ spectra these models were not sensitive enough to fix simultaneously the values of the temperature and the radial expansion velocity without ambiguities, the precision of the present data allows to further restrict the parameter values. The spectrum is best described with a temperature $T=184 \mathrm{MeV}$ and an average transverse flow velocity of $\langle\beta\rangle=0.21$. The fits are not very sensitive to the baryonic chemical potential because baryon resonances contribute

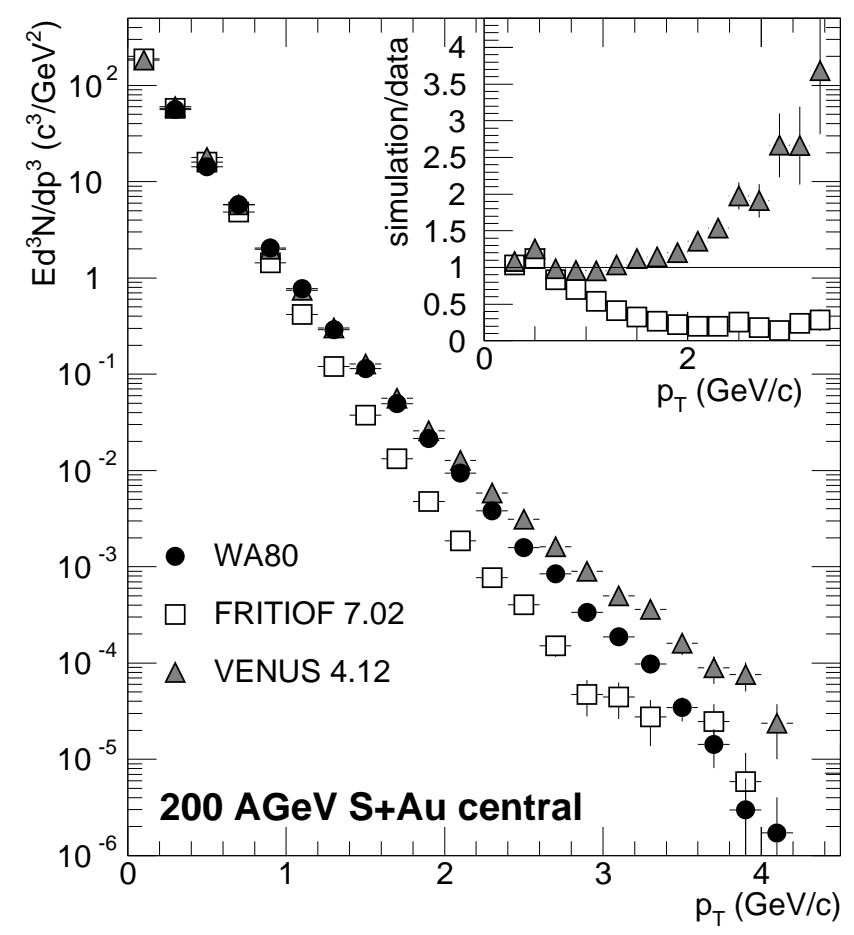

Fig. 13. Comparison of the $\pi^{0}$ invariant yields per event of central (7.7\%) $200 \mathrm{GeV} \mathrm{S}+\mathrm{Au}$ data with results from string models VENUS [12] and FRITIOF [1] both filtered with the experimental acceptance. The inset shows the ratios of the model predictions to the experimental data. 


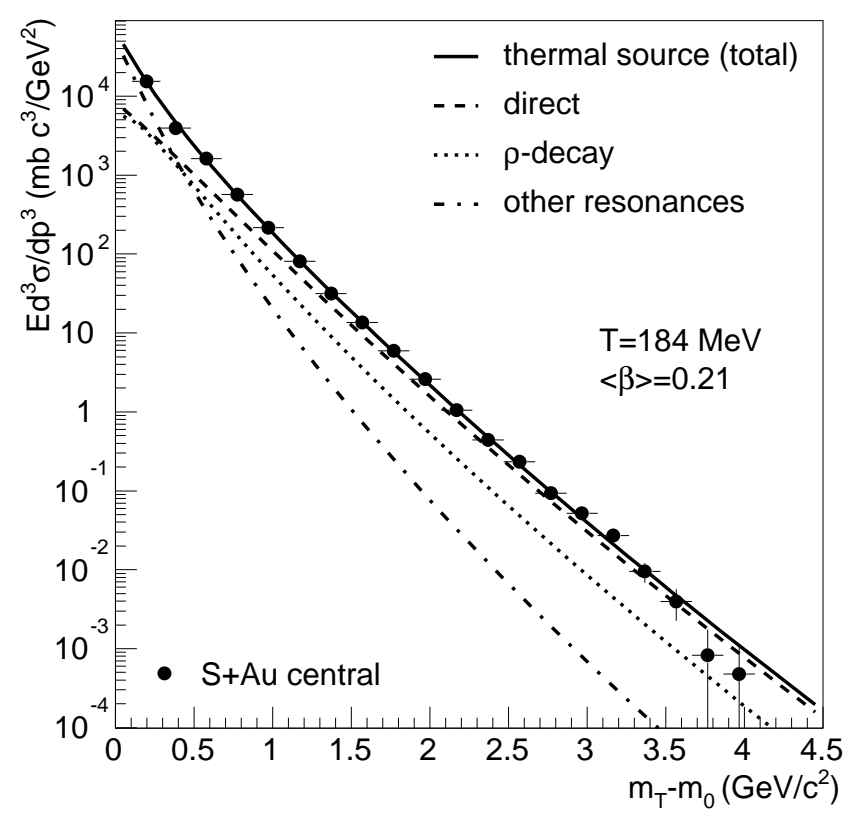

Fig. 14. Fits to the $\pi^{0} m_{T}$ distribution of central $\left(7.7 \%\right.$ of $\left.\sigma_{\mathrm{m} b}\right) 200$ $\mathrm{GeV} \mathrm{S}+\mathrm{Au}$ collisions within a thermodynamic model including resonance decays 42 ].

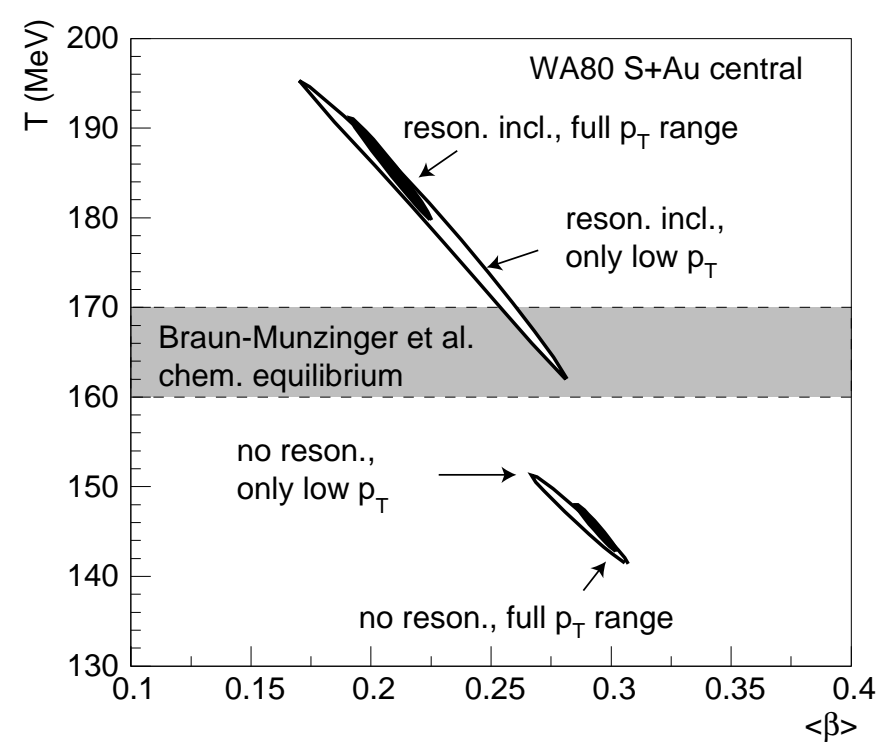

Fig. 15. Confidence regions (1 standard deviation) for the fit parameters of a thermodynamic model including resonance decays [42] in central $\left(7.7 \%\right.$ of $\left.\sigma_{\mathrm{m} b}\right) 200 \mathrm{GeV} \mathrm{S}+\mathrm{Au}$ collisions. The horizontal band indicates the chemical temperature values extracted from particle ratios [49].

mostly at very low $p_{T}$ where the experimental coverage vanishes. This can be seen from Fig. 14, where the total yield is broken down into the direct thermal pions (dashed line), the contribution from $\rho$ decays (dotted line) and all other resonances (dash-dotted line).

Fig. 15 shows 1-sigma-confidence regions in the $(T,\langle\beta\rangle)$ parameter plane for these fits. Calculations have been performed under the following conditions:
1. Including all resonances using

(a) the full experimental $m_{T}$-range (solid area in the upper part of Fig. 15) and

(b) only data for $m_{T}<1.6 \mathrm{MeV} / c^{2}$ (outline in the upper part of Fig. 15)

and

2. including only the direct pions using the same $m_{T}$ regions (a) and (b) as above (solid area and outline in the lower part of Fig. 15).

A fit to the entire $m_{T}$ range with the full model constrains the temperature to the range $T=180-190 \mathrm{MeV}$, a fit to the restricted $m_{T}$ range yields essentially the same optimal parameters $(T=178 \mathrm{MeV})$, but relaxes the constraints - still the $1-\sigma$ range of the temperature is above $160 \mathrm{MeV}$. The $2-\sigma$ confidence region is essentially unbound towards lower temperatures when using only data for $m_{T}<1.6 \mathrm{MeV} / c^{2}$, one can, however, see that such fits for lower temperatures are in contradiction with the data at higher $m_{T}$. Fig. 16 shows the ratio of fits to the experimental data for the optimum value $(T=178 \mathrm{MeV})$ and constrained fits for $T=140 \mathrm{MeV}$ and $T=120 \mathrm{MeV}$. It can be seen that the fits for lower temperatures overpredict the cross section at higher $m_{T}$. In the presence of additional hard scattering contributions the experimental data would still provide an upper bound for the hydrodynamical contribution. Therefore the full $m_{T}$ range may be used to estimate a lower bound of the temperature parameter.

It is interesting to note that fits ignoring the resonance contributions would indicate much lower temperatures and also slightly higher flow velocities than those of the full calculation. In this case the temperature and flow velocity are rather close to the values given in 47] for $\mathrm{S}+\mathrm{S}$ collisions $(T \approx 140 \mathrm{MeV}$, $\langle\beta\rangle=0.24-0.28$ ) 6 Similar numbers are given in [48]. One should keep in mind, however, that there is no good reason to exclude the resonance decays, and that our results for the full calculation are not consistent with the values in [47,48].

While the calculation with resonances is clearly more reasonable than the one without, there are still further uncertainties related to the model assumptions. Especially the choice of one temperature to characterize both chemical freeze-out of all resonances and thermal freeze-out of the pions is questionable. The chemical freeze-out temperature of the $\rho$ meson as a resonance appearing in $\pi \pi$ scattering might be closely related to the thermal freeze-out temperature of the pions. However, this is most likely not true for other particles like e.g. the $K^{\star}$. It is therefore necessary to add information from other measurements, e.g. relative particle abundancies or interferometry measurements.

Due to the finite value of the expansion velocity, i.e. an energy sharing between random thermal and ordered collective motion, the resulting temperature is now smaller than the slope parameter obtained from the exponential fits in Fig. 6 . The temperature of $184 \mathrm{MeV}$ can be compared to the value of $T=160-170 \mathrm{MeV}$ extracted from relative particle abundancies [49].

\footnotetext{
${ }^{6}$ The $\mathrm{S}+\mathrm{S}$ data in the present publication yield similar results to the $\mathrm{S}+\mathrm{Au}$ data, their statistical uncertainty is, however, much larger so that they have not been considered further.
} 


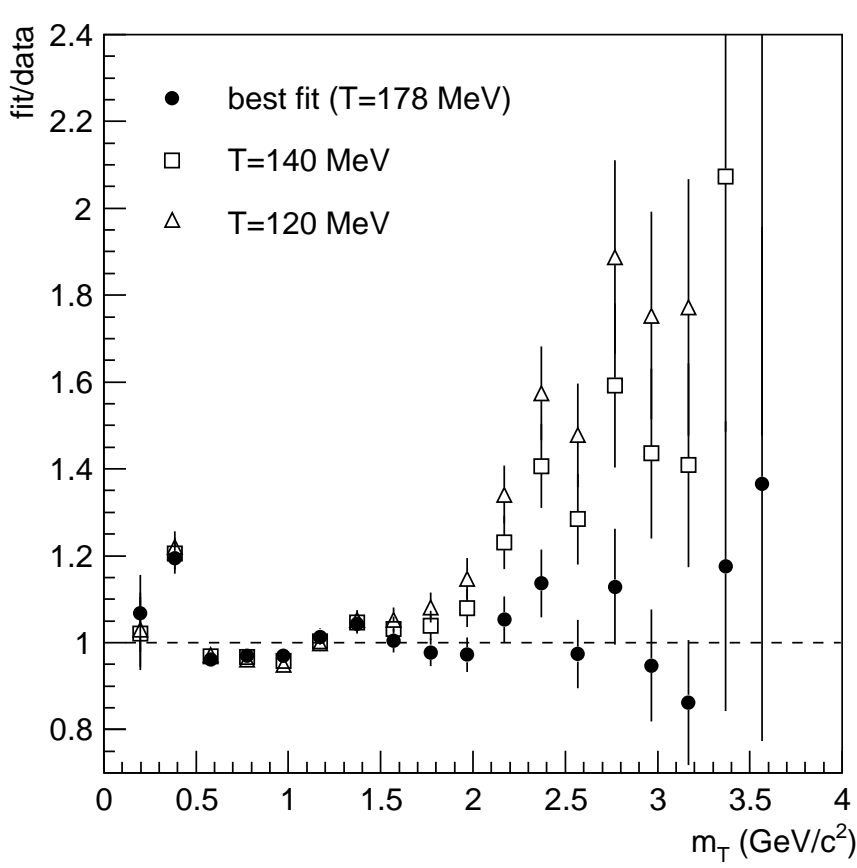

Fig. 16. Ratio of fits of a thermodynamic model including resonance decays [42] to data for central $\left(7.7 \%\right.$ of $\left.\sigma_{\mathrm{mb}}\right) 200 \mathrm{GeV} \mathrm{S}+\mathrm{Au}$ collisions for different temperatures.

\section{Summary}

We have discussed transverse momentum spectra of neutral pions produced in various systems. The extracted local slopes as well as the power-law fits to the pp and $\mathrm{AB} p_{T}$ spectra unambiguously demonstrate concave distributions for all systems and impact parameter selections. The shapes of the spectra can be well characterized by two parameters, e.g. the inverse slope at a given $p_{T}\left(T=T_{1}\right)$ and the linear variation of the slope parameters $\left(\Delta T=T_{2}-T_{1}\right)$. While $\Delta T$ (the "slope of the local slope") and thereby the curvature are very similar in all systems, there is a strong increase of the inverse slope parameters and of $\left\langle p_{T}\right\rangle$ in going from pp to heavy ion collisions and a continuing, but more subtle growth with the size of the interaction zone.

The comparison to event generators shows, that they still fail to describe all subtle features observed in the data if measured over a large dynamic range. The FRITIOF model shows large discrepancies, VENUS 4.12 with its phenomenological rescattering provides a better, yet still unsatisfactory description. A hydrodynamically motivated calculation with two fitted parameters agrees very nicely with the data. Within such a calculation both transverse flow and decay of long-lived resonances appear to be of importance for the interpretation of measured transverse momentum spectra. The fit applied to the data in the present paper favours comparably large freeze-out

\footnotetext{
${ }^{7}$ Corrections for the influence of a shift of the effective center-ofmass on the transverse momentum spectra measured as a function of centrality in asymmetric heavy-ion collisions have not been considered here, although they might - within certain thermal models - account for part of the centrality dependence of the slope parameters.
}

temperatures and low flow velocities. As such large temperatures might require high pion phase space densities, it has to be investigated whether they may be interpreted as freeze-out temperatures or whether the model used here with the assumption of local thermal equilibrium may be not applicable.

The spectra are broadened when going from $\mathrm{pp}$ to $\mathrm{AB}$ collisions. At first sight, ratios of the type $\mathrm{pA} / \mathrm{pp}, \mathrm{AB} / \mathrm{pp}$, central/peripheral, etc. seem to isolate the existence of strong high $p_{T}$ phenomena. A closer look at the individual changes of the spectral shapes, however, allows to trace the increasing ratios with increasing $p_{T}$ back to the differences in the overall shape of the spectra which necessarily leads to large effects in the cross section at large $p_{T}$. The difference in the slopes over the full $m_{T}$ range may be taken as a hint for non-trivial nuclear effects observed in these collisions. The observed variations may, in the framework of thermodynamics, be taken as an indication of higher temperature and/or a possible stronger collective expansion of the system in heavy ion collisions compared to pp. Likewise, in the picture of string models, it may suggest a stronger amount of rescattering of produced particles. Another possible conclusion would be to call for different mechanisms, i.e. string formation and decay as sufficient for pp collisions, but local thermodynamic equilibrium and hydrodynamic expansion in heavy ion collisions.

Common to all models, even the most conservative ones, is the need to either incorporate large amounts of initial- and final-state scattering, or to assume high temperature and pressure build up. Therefore, they imply a dense and strongly interacting system, i.e. conditions which are favorable to eventually reach thermal equilibrium.

This work was supported jointly by the German BMBF and DFG, the U.S. DOE, the Swedish NFR, the Humboldt Foundation, the International Science Foundation under Contract N8Y000, the INTAS under Contract INTAS-93-2773, ORISE, and the Dutch FOM. ORNL is managed by Lockheed Martin Energy Systems under contract DEAC05-84OR21400 with the U.S. Department of Energy. We also like to thank the accelerator divisions of CERN for their excellent work.

\section{References}

1. R. Hagedorn, Suppl. Nuovo Cimento 3 (1965) 147-186.

2. B. Alper et al., Nucl. Phys. B100 (1975) 237-290.

3. F.W. Büsser and et al, Phys. Lett. 46B (1973) 471-476.

4. F.W. Büsser et al., Nucl. Phys. B106 (1976) 1-30.

5. M. A. Faessler, Phys. Rep. 115 (1984) 1-91.

6. W.M. Geist et al., Phys. Rep. 197 (1990) 263-374.

7. J.W. Cronin et al., Phys. Rev. D11 (1975) 3105-3123.

8. A. Krzywicki et al., Phys. Lett. B85 (1979) 407-416.

9. M. Lev and B. Petersson, Z. Phys. C21 (1983) 155-161.

10. H. Sorge, H. Stöcker, and W. Greiner, Nucl. Phys. A498 (1989) $567 \mathrm{c}-576 \mathrm{c}$.

11. B. Andersson, G. Gustafson, and H. Pi, Z. Phys. C57 (1993) 485494.

12. K. Werner, Phys. Rep. 232 (1993) 87-299.

13. K.S. Lee and U. Heinz, Z. Phys. C43 (1989) 425-429.

14. E. Schnedermann et al., Phys. Rev. C48 (1993) 2462-2475.

15. L. Landau, Proc. Acad. Sci., USSR (Phys. Series) 17 (1953) 51.

16. R. Santo et al., Nucl. Phys. A 566 (1994) 61c-68c 
17. R. Albrecht et al., WA80 Collaboration, Phys. Lett. B361 (1995) 14-20.

18. R. Albrecht et al., Phys. Rev. Lett. 76 (1996) 3506

19. F. Berger et al., Nucl. Instr. Meth. A321 (1992) 152-164.

20. H. Baumeister et al., Nucl. Instr. Meth. A292 (1990) 81-96.

21. R. Albrecht et al., WA80-Collaboration, Z. Phys. C47 (1990) 367-375.

22. R. Albrecht et al., Nucl. Instr. Meth. A276 (1989) 131-139.

23. T. C. Awes et al., Nucl. Instr. Meth. A279 (1989) 479-502.

24. R. Albrecht et al., WA80-Collaboration, Phys. Rev. C44 (1991) 2736-2752.

25. R. Brun et al. GEANT3, CERN Data Handling Division. DD/EE/84-1, 1987.

26. J. Bächler et al., NA35 Collaboration, Phys. Rev. Lett. 72 (1994) 1419-1422.

27. G. Bocquet et al., UA1-Collaboration, Phys. Lett. B366 (1996) 434.

28. R. Hagedorn, CERN-TH. 3684, 1983.

29. E.W. Beier et al., Phys. Rev. D18 (1978) 2235-2238.

30. C. DeMarzo et al., NA24 Collaboration, Phys. Rev. D36 (1987) $16-20$.

31. H.R. Schmidt and J. Schukraft, J. Phys. G19 (1993) 1705-1796.

32. D. Antreasyan et al., Phys. Rev. D19 (1979) 764.

33. J. Povlis et al., Phys. Rev. Lett. 51 (1983) 967-970.

34. T. Åkesson et al., NA34-Collaboration, Z. Phys. C46 (1990) 361367.

35. D. Chaney et al., Phys. Rev. D19 (1979) 3210-3221.

36. F. Becattini, Z. Phys. C69 (1996) 485-492.

37. K. Geiger, Phys. Rev. D46 (1992) 4965-4985.

38. K. Geiger, Phys. Rev. D46 (1992) 4986-5005.

39. K. Werner and J. Aichelin, Phys. Rev. C52 (1995) 1584-1603.

40. H. Sorge, Phys. Rev. C52 (1995) 3291-3314.

41. E. Schnedermann, Phys. Rev. C50 (1994) 1675.

42. U.A. Wiedemann and U. Heinz, Phys. Rev. C56 (1997) 32653286.

43. R. Albrecht et al., WA80-Collaboration, Z. Phys. C55 (1992) 539-548.

44. M. Aguilar-Benitez et al., NA27-Collaboration, Z. Phys. C50 (1991) 405-426.

45. H. Grässler et al., Nucl. Phys. B132 (1978) 1-14.

46. J. Sollfrank et al., Z. Phys. C52 (1991) 539.

47. I.G. Bearden et al., Phys. Rev. Lett. 78 (1997) 2080-2083.

48. J. Sollfrank et al., Mass number scaling in ultra-relativistic nuclear collisions from a hydrodynamical approach, preprint nuclth/9801023 and BI-TP 97/55.

49. P. Braun-Munzinger et al., Phys. Lett. B365 (1996) 1-6. 